\title{
Mineração
}

\section{Modelamento matemático de peneiramento vibratório (Parte 1): dimensionamento clássico}

\author{
José Aurélio Medeiros da Luz \\ Universidade Federal de Ouro Preto - UFOP \\ Escola de Minas - Departamento de Engenharia de Minas - DEMIN \\ E-mail: jaurelio@demin.ufop.br
}

Simão Célio de Carvalho

Engenheiro de Minas, Sempre Viva Mineração - Construções e Transportes Mestrando em Engenharia Mineral - DEMIN/UFOP

E-mail:simao@sviva.com.br

\section{Resumo}

A partição do fluxo em peneira é fortemente dependente da distribuição de probabilidade de passagem de partículas por suas aberturas. Essa probabilidade é influenciada pelas condições de operação e pela relação entre o tamanho de partícula e abertura. Desenvolveu-se um sistema (SimPeneira) para dimensionar peneiras, utilizando o método clássico de dimensionamento (adequadamente modificado), e simular diversos cenários de peneiramento industrial, quantificando o impacto de parâmetros operacionais. Esse artigo descreve a primeira parte de tal sistema, abordando o dimensionamento clássico de peneiras vibratórias e revolventes.

Palavras-chave: dimensionamento, simulação, granulometria, peneiramento.

\begin{abstract}
Flow splitting in screens is strongly dependent on probability distribution of particles passage through the screen openings. This probability is influenced by operational conditions and the particle size to opening ratio. A software system called SimPeneira was developed to perform sizing of screen using the classical sizing procedure (with changes) and also to simulate several scenarios in industrial screening, quantifying the impact of operational parameters. This article describes the first part, embracing the sizing pack.
\end{abstract}

Keywords: screen sizing, simulation, and screening. 


\section{Introdução}

Peneiramento é o processo de separação de um material granular não coeso em duas ou mais diferentes classes de tamanho de partículas, mediante uma ou mais superfícies vazadas com aberturas de dimensões definidas. Essa classificação por tamanho, portanto, é feita por barreira mecânica (nos processos de classificação em correntes fluidas, a barreira é fluidodinâmica). É um processo do tipo "passa/não passa" e as barreiras são constituídas pelos fios da malha. Em geral, peneira refere-se à superfície tecida com fios espaçados regularmente e crivos àquela feita de chapa perfurada.

Os objetivos usuais do peneiramento industrial são: evitar a entrada de partículas menores, ou subtamanho (undersize) no equipamento a jusante; evitar que o sobretamanho (oversize) passe para os estágios subseqüentes; bitolar adequadamente o material para aumento da eficiência das operações a jusante (formação de fluxo paucidisperso) ou para adequar um produto a especificações.

A quantificação da partição do fluxo de alimentação é fortemente dependente da distribuição de probabilidade de passagem de cada classe granulométrica pelas aberturas das telas, probabilidade essa influenciada pelas condições de operação e pela relação entre o tamanho de partícula e tamanho de abertura efetiva.

O objetivo desse trabalho é apresentar o módulo de dimensionamento de peneiras de um modelo computacional para simulação de peneiramento (sistema SimPeneira). Esse sistema é capaz de simular diversos cenários de peneiramento, bem como dimensionar peneiras segundo o procedimento clássico baseado no método Allis-Chalmers modificado.

\section{Materiais e métodos}

O recurso material necessário ao desenvolvimento do módulo de dimensionamento do sistema computacional consistiu em microcomputador, ambiente integrado de desenvolvimento
Delphi (versão 7), gerenciador de banco de dados Microsoft Access e o software de tratamento estatístico EasyPlot.

\subsection{Modelo de dimensionamento clássico de peneiras vibratórias}

A grande maioria dos formalismos para dimensionamento de peneiras vibratórias e revolventes constantes na literatura é variante do chamado método AllisChalmers. Esse método consiste em recorrer a uma curva de capacidade unitária padrão, em função da abertura efetiva da malha, $\boldsymbol{a}$, e afetar o valor lido por fatores de correção devidos aos desvios das condições padronizadas, utilizadas pelos fabricantes quando do estabelecimento da curva de capacidade unitária padrão.

Um método independente é o de Menne (Menne, 2002), para cálculo de área de peneiras vibratórias, a qual pode ser expressa por:

$$
S=\left\{\left[\frac{(1-s)-m}{7,8 \times a \times f_{a}^{2}}\right] \times \ln \left[\frac{(1-s)-m}{\varepsilon \times(1-s)-m}\right]+K\right\} \times Q_{a}
$$

Onde:

$Q_{a}$ - vazão mássica de alimentação [t/h].

$\boldsymbol{m}$ - fração de material menor que a metade de $\boldsymbol{a}$, no fluxo de alimentação do deck [-].

$\boldsymbol{s}$ - fração de material maior que $\boldsymbol{a}$, na alimentação do deck [-].

$f_{a}$ - fração de área aberta da peneira [-].

$\boldsymbol{\varepsilon}$ - eficiência de remoção de finos [-].

$\boldsymbol{K}$ - parâmetro dado por:

$$
\left\{\begin{array}{l}
K=0 ; \text { se } \quad \text { a }>400 \mathrm{~mm} \\
K=\frac{2,5 \times(424-a)}{10000} ; \text { se } \quad \mathrm{a} \leq 400 \mathrm{~mm}
\end{array}\right.
$$

Contudo o módulo de dimensionamento do SimPeneira utilizou o método AllisChalmers, em face de seu uso generalizado. Para se evitarem o uso de tabelas no programa e a necessidade do uso de rotinas de interpolação (por vezes indutora de erros), foi realizada uma criteriosa análise de regressão com os valores tabelados para dimensionamento pelo método Allis-Chalmers (Faço, 1994, Chaves e Peres, 1999). A equação que dá a área requerida para o peneiramento, pelo método clássico, é:

$$
S=\frac{\frac{Q_{a}}{\rho_{a p}} \times F_{p}}{Q_{b a s} \times \prod k_{i}}
$$

onde:

$Q_{a}$ - vazão mássica de alimentação[t/h].

$\boldsymbol{F}_{p}$ - fator de projeto [-].

$Q_{b a s}^{p}$ - capacidade específica do peneiramento $\left[\left(\mathrm{m}^{3} / \mathrm{h}\right) / \mathrm{m}^{2}=\mathrm{m} / \mathrm{h}\right]$.

$\Pi \boldsymbol{k}_{i}$ - "produtório" dos vários fatores de correção, $\mathrm{k}_{\mathrm{i}}[-]$.

$\boldsymbol{\rho}_{a p}$ - massa específica aparente do material $\left[\mathrm{t} / \mathrm{m}^{3}\right]$.

Naturalmente, é necessário possuir, ou a análise granulométrica, ou os parâmetros da distribuição estatística que a descreva.

A capacidade básica de peneiramento, $\boldsymbol{Q}_{b a s}$ (em função da abertura em milímetros), é dada por: 


$$
Q_{\text {bas }}=0,36423 \times \sqrt{a^{2}+251,28 \times a}
$$

A determinação do fator $\boldsymbol{k}_{1}$, o qual é o fator de correção em função do "tamanho metade" ( $\boldsymbol{m}$, dado em milímetros), utiliza a seguinte fórmula de regressão:

$$
k_{1}=\sqrt{4,815 \times \frac{m}{1000}^{2}+0,193}
$$

O fator de correção, $\boldsymbol{k}_{2}$, para a fração retida (fator de grossos) pode ser calculado, utilizando-se a seguinte fórmula de regressão:

$$
k_{2}=-2,72 \times(1-\exp (-0,08 \times(90,1-s)))+3,7
$$

Se os parâmetros estatísticos da distribuição granulométrica na alimentação forem conhecidos, as frações de material abaixo do tamanho metade $(\boldsymbol{m})$ e a de grossos $(\boldsymbol{s})$ podem ser calculadas substituindo o argumento da equação por $\boldsymbol{a} / \mathbf{2} \mathrm{e}$ por $\boldsymbol{a}$, respectivamente.

O fator de tipo de abertura, $\boldsymbol{k}_{3}$, é calculado do seguinte modo: para malha quadrada $\boldsymbol{k}_{3}=\mathbf{1}$; para malha redonda $\boldsymbol{k}_{3}=\mathbf{0 , 8}$. Caso a malha seja retangular, $\boldsymbol{k}_{3}$ será dado pela seguinte fórmula:

$$
k_{3}=0,274 \times\left(1-\exp \left(-0,738 \times\left(k_{d}-1\right)\right)\right)+1
$$

onde: $\boldsymbol{k}_{\boldsymbol{d}}$ é a relação entre os lados da abertura $\left(\boldsymbol{d}_{\boldsymbol{I}} / \boldsymbol{d}_{2}\right)$.

O fator de ajuste para o formato das partículas é dado pelos critérios seguintes: $\boldsymbol{k}_{\mathbf{4}}=\boldsymbol{1}$ para partículas cúbicas e $\boldsymbol{k}_{4}=0,9$ para as lamelares.

O fator de eficiência da abertura, $\boldsymbol{k}_{5}$, é igual a 1,0 para peneiramento a seco ou calculado do seguinte modo, se o peneiramento for a úmido (para abertura da peneira dada em $\mathrm{mm}$ ):

$$
k_{5}=-0,04 \times \sqrt{a-3,37}+1,407
$$

O fator de umidade, $\boldsymbol{k}_{\boldsymbol{\sigma}}$, é calculado do seguinte modo (onde $\boldsymbol{u}$ representa o percentual de umidade):

- $\operatorname{Para} 6 \%<\mathrm{u}<9 \%: \boldsymbol{k}_{6}=0,75$.

- Para $3 \%<\mathrm{u}<6 \%: \boldsymbol{k}_{6}=0,85$.

- Para peneiramento a úmido ou para $u<3 \%: \boldsymbol{k}_{6}=1$.

$\mathrm{O}$ fator de área efetiva, $\boldsymbol{k}_{7}$, é calculado em função do deck em questão, do seguinte modo: para primeiro deck $\boldsymbol{k}_{7}=\mathbf{0 , 9}$; para segundo deck $\boldsymbol{k}_{7}=\mathbf{0 , 8}$; e para terceiro deck $\boldsymbol{k}_{7}=\mathbf{0 , 7}$.

O fator de área aberta, $\boldsymbol{k}_{\boldsymbol{g}}$ (em função da abertura efetiva da peneira,expressa em milímetros), é calculado do seguinte modo:

$$
k_{8}=\frac{f_{a}}{50}=\frac{34 \times(1-\exp (-0,05 \times(a-1,9)))+40}{50}
$$

Com se vê do método apresentado, não há correções para a inclinação da peneira e, portanto, os ábacos e tabelas Allis-Chalmers pressupõem a adoção dos valores típicos recomendados pelo fabricante. A partir desses dados tabelados
(Chaves \& Peres, 1999), a inclinação, em graus, usualmente recomendada depende da abertura da tela e pode ser calculada pela seguinte equação (em função da abertura a em milímetros):

$\alpha=4,82 \times \ln (a)-2,4$

Igualmente, o método Allis-Chalmers não explicita a influência da velocidade angular do sistema oscilatório. $\mathrm{O}$ valor preconizado depende também da abertura e pode ser obtido (em rpm) pela seguinte equação (em função da abertura a também em milímetros):

$N=1695,4 \times(a)^{-0,1651}$

Naturalmente, tanto $\boldsymbol{\alpha}$ quanto $\boldsymbol{N}$, obtidos pelas respectivas equações, são apenas valores costumeiros, já que, em certa medida, são parâmetros de escolha do projetista.

\subsection{Modelo de dimensionamento de peneiras revolventes}

Nos próximos parágrafos, descreve-se o procedimento equivalente para dimensionamento de peneiras revolventes (trômeis). Para o cálculo da área requerida deve-se utilizar a equação a seguir:

$\boldsymbol{S}=\boldsymbol{\pi} \times \boldsymbol{D} \times \boldsymbol{L}$

onde: $\boldsymbol{D}$ - Diâmetro efetivo do trômel [m].

$\boldsymbol{L}$ - Comprimento efetivo do trômel [m].

O tempo de residência das partículas dentro do trômel (em segundos) pode ser previsto, utilizando-se a equação a seguir (Porter, 1984):

$\tau=\frac{0,23 \times L}{N_{t r}^{0,9} \times D \times \operatorname{tg}(\alpha)}$

onde: $\boldsymbol{N}_{t r}$ - velocidade de rotação do trômel [rpm].

$\boldsymbol{\alpha}$ - inclinação do eixo longitudinal do trômel [ $\left.{ }^{\circ}\right]$.

O comprimento do trômel é dado pela equação (Gomide, 1983) apresentada a seguir:

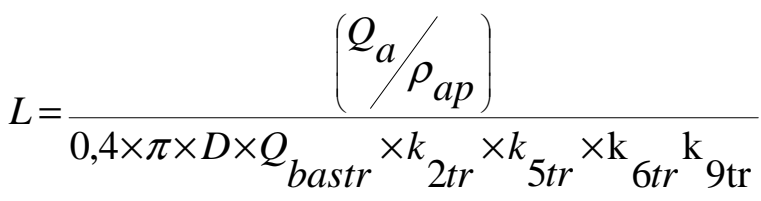

Onde a capacidade básica de peneiramento por $\mathrm{m}^{2}$ de tela do trômel, $\boldsymbol{Q}_{\text {bastr }}$, é dada pela equação de regressão seguinte:

$Q_{\text {bastr }}=1,06 \times \ln (0,562 \times$ a $)$

onde $\boldsymbol{a}$ é a abertura da tela, dada em milímetros.

O fator de correção para a fração retida (fator de grossos), $\boldsymbol{k}_{2 t r}$, pode ser calculado utilizando-se a seguinte fórmula:

$k_{2 t r}=-2,5203 \times s^{3}+2,2569 \times s^{2}-0,8943 \times s+1,1623$

O fator de eficiência do peneiramento $\boldsymbol{k}_{5 t r}$ é calculado do 
seguinte modo, em função da eficiência de remoção de finos:

$k_{5 t r}=-0,000029 \times \varepsilon^{3}+0,0064 \times \varepsilon^{2}-0,4875 \times \varepsilon+14,145$

O fator de inclinação, $\boldsymbol{k}_{9 t r}$, por sua vez, é calculado do seguinte modo (em função da inclinação, $\boldsymbol{\alpha}$, em graus):

$k_{9 t r}=0,01976 \times \alpha^{2}-0,3974 \times \alpha+2,51$

O fator de umidade e o tipo de abertura de passagem, $\boldsymbol{k}_{\boldsymbol{\sigma t r}}$, são dado por:

- Peneiramento a úmido e aberturas redondas: $\boldsymbol{k}_{\boldsymbol{\sigma t r}}=1$.

- Peneiramento a úmido e aberturas quadradas: $\boldsymbol{k}_{\boldsymbol{\sigma t r}}=1,2$.

- Peneiramento a seco e aberturas redondas: $\boldsymbol{k}_{6 t r}=1,6$.

- Peneiramento a seco e aberturas quadradas: $\boldsymbol{k}_{\boldsymbol{\sigma t r}}=1,9$.

\section{Resultados e discussão}

A interface do módulo de dimensionamento clássico é apresentada na Figura 1. A tela apresentada, nessa figura, mostra os dados do manual de britagem da Faço (Faço, 1994), os quais foram lançados, a título de exemplificação da adequação do algoritmo implementado. Os resultados obtidos podem ser observados na Figura 2.

Comparando-se a área calculada pelo programa $\left(6,8 \mathrm{~m}^{2}\right)$ com o resultado do manual de britagem $\left(6,4 \mathrm{~m}^{2}\right)$, vê-se que houve precisão razoável. Deve-se registrar que o desvio observado poderia ser diminuído com ulteriores melhorias nas equações de regressão. Entretanto isso não se justificaria, pois os próprios valores tabelados na literatura são cifras aproximadas e não exatas.

\section{Conclusões}

Embora o modelo computacional (sistema SimPeneira) tenha sido concebido primacialmente para simulação probabilística de peneiramento vibratório, a inserção de um módulo de dimensionamento de peneiras, baseado na análise de regressão do procedimento clássico, possibilita sua utilização para o dimensionamento direto de uma planta de peneiramento, piloto ou industrial.

A utilização do módulo de simulação probabilística, partindo-se do formalismo de Mogensen (Mogensen, 1965, Kelly \& Spottiswood, 1982), para o dimensionamento indireto, apresenta a vantagem de levar em conta parâmetros tais como: a abertura, comprimento e inclinação da peneira, velocidade do fluxo do material, área efetiva de peneiramento, freqüência e amplitude das vibrações. Tal módulo será objeto da segunda parte desse trabalho, intitulado: "Modelamento matemático de peneiramento vibratório (Parte 2): simulação".

Em qualquer das instâncias, o programa também pode servir como excelente suporte didático nos cursos regulares de engenharias de minas, metalúrgica e química.

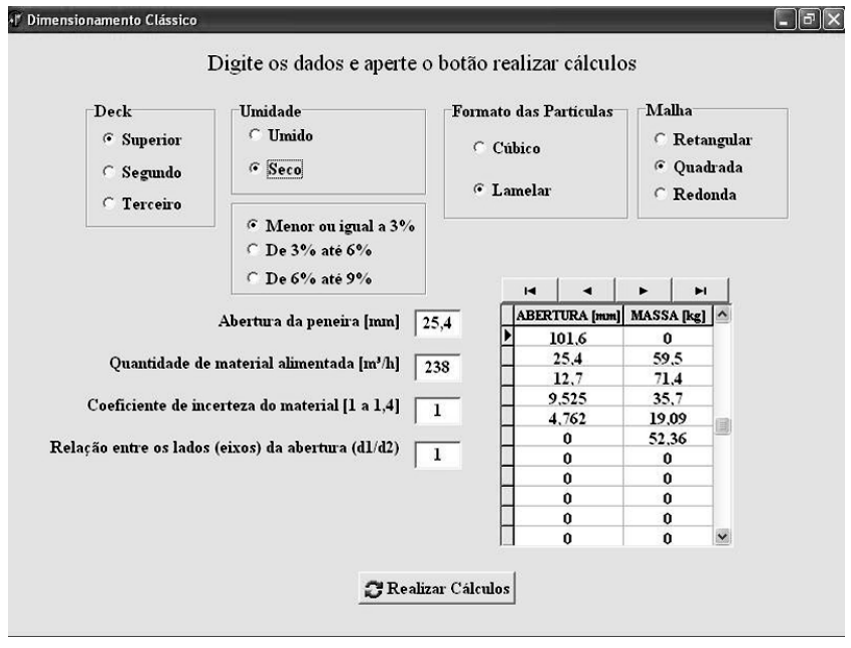

Figura 1 - Módulo de dimensionamento do programa SimPeneira.

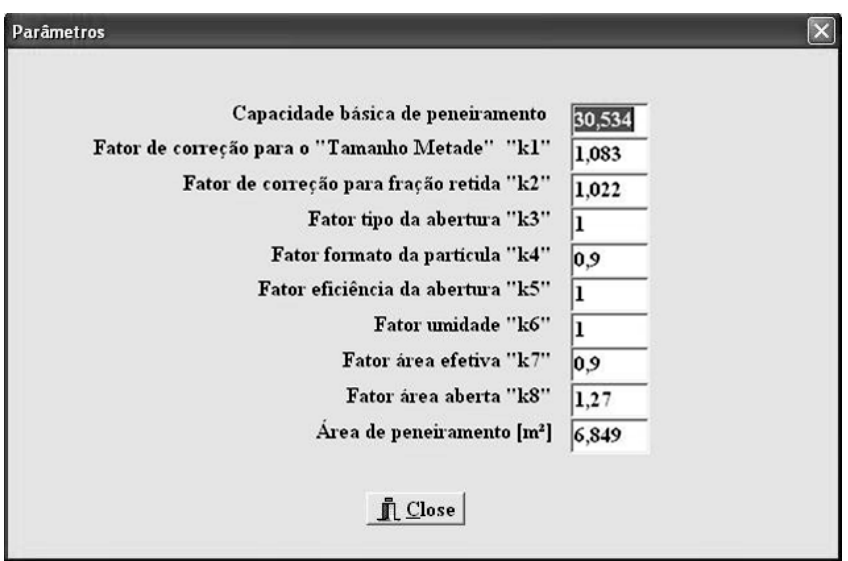

Figura 2 - Resultados do SimPeneira módulo de dimensionamento.

\section{Agradecimentos}

Os Autores expressam seu reconhecimento à CAPES, pelo suporte financeiro, e ao CEFETOP, pela liberação do laboratório para a realização dos ensaios de peneiramento.

\section{Referências Bibliográficas}

CHAVES, A. P., PERES, A. E. C. Teoria e prática do tratamento de minérios - britagem, peneiramento e moagem. 1. ed. São Paulo: Signus, 1999. v. 3. 238p.

FAÇO. Manual de britagem da Faço. São Paulo: Allis Mineral Systems, 1994.

GOMIDE, R. Operações unitárias. São Paulo: Edição do Autor 1983. v.1. 293p.

KELLY, E. G., SPOTTISWOOD, D. J. Introduction to mineral processing. New York: John Wiley, 1982.

MEMME, D. Screening Solids and Slurries. Disponível em: <http:// membres.iinet.net.au/ menne/screen2.htm>. Acesso em: 31/05/2002.

MOGENSEN, F. A new screening method of screening granular materials. The Quarry Managers' Journal. p. 409-414. Oct., 1965.

PORTER, H. F. et alii. Solid drying and gas solid systems. In: PERRY, R. H., GREEN, D. Perry's Chemical Engineers'Handbook. New York: MacGraw-Hill, 1984.

Artigo recebido em 20/06/2004 e aprovado em 03/01/2005. 